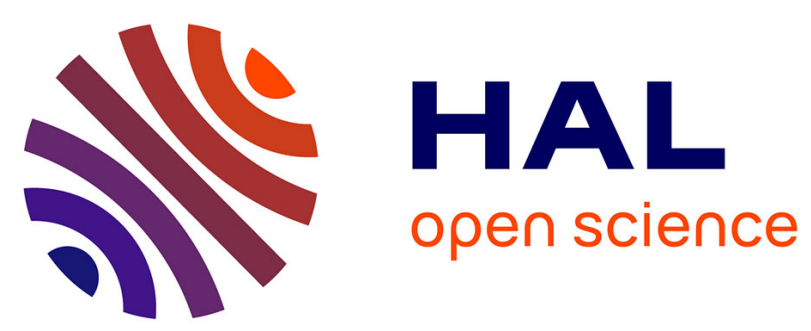

\title{
Wadi Iram: un lieu du culte et de rassemblement des tribus arabes dans l'antiquité
}

Saba Farès-Drappeau

\section{To cite this version:}

Saba Farès-Drappeau. Wadi Iram: un lieu du culte et de rassemblement des tribus arabes dans l'antiquité. ARAM Periodical, 1996, 8 (2), pp.269 - 283. 10.2143/ARAM.8.2.2002199 . halshs01728375

\section{HAL Id: halshs-01728375 \\ https://shs.hal.science/halshs-01728375}

Submitted on 24 Sep 2021

HAL is a multi-disciplinary open access archive for the deposit and dissemination of scientific research documents, whether they are published or not. The documents may come from teaching and research institutions in France or abroad, or from public or private research centers.
L'archive ouverte pluridisciplinaire HAL, est destinée au dépôt et à la diffusion de documents scientifiques de niveau recherche, publiés ou non, émanant des établissements d'enseignement et de recherche français ou étrangers, des laboratoires publics ou privés. 


\title{
WĀDĪ IRAM: UN LIEU DU CULTE ET DE RASSEMBLEMENT DES TRIBUS ARABE DANS L'ANTIQUITÉ. LES PREMIERS RÉSULTATS DE LA MISSION ÉPIGRAPHIQUE 1997
}

\author{
SABA FARÈS-DRAPPEAU
}

En février 1996, une modeste exploration épigraphique a été menée dans la région immédiate de Wādī Iram en Jordanie du sud avec un financement personnel. L'équipe était composée de M. Fawzi ZaYADine, du Département des Antiquités de Jordanie, de M. Frédéric AlPI, alors pensionnaire scientifique à l'Institut Français d'Archéologie du Proche-Orient/Beyrouth, de M. Houssein ABu AL-Hassan de l'Université du roi Séoud à Riyad et de moi-même. L'exploration s'est faite sous les auspices de l'Institut Français d'Archéologie du Proche-Orient avec le concours du Département des Antiquités de Jordanie. Durant la période du 17 au 24 février 1996 nous avons enregistré quelque 150 inscriptions inédites. Ces résultats ont encouragé l'Institut Français d'Archéologie du Proche-Orient et le Département des Antiquités de Jordanie à s'engager dans un programme de recherche épigraphique dans cette région. Ce programme associe l'Université du Yarmouk/Département d'épigraphie et l'Université du roi Séoud à Riyad. En juin 1997 nous sommes retournés pour une période de quatre semaines, mais cette fois avec une aide matérielle importante fournie par l'IFAPO. Le nombre des inscriptions enregistrées est de 535 textes du type thamoudéen. L'étude des résultats de la mission est en cours, ici sont présentées uniquement la description du site et quatre inscriptions à titre d'exemple.

Le Wādī Iram se trouve dans le sud de la Jordanie, à environ $325 \mathrm{~km}$ au sud d'Amman, $40 \mathrm{~km}$ au nord de 'Aqaba et $29 \mathrm{~km}$ du croisement 'Aqaba-Dīsseh. Le village d'Iram est habité par des bédouins sédentarisés de la tribu de

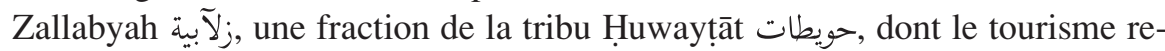
présente la principale ressource.

Géologiquement, le Wādī Iram est le prolongement naturel du bouclier arabonubien, dont le Hiiǧãz constitue l'extrémité la plus au sud. Les arabes nomades ont trouvé refuge dans ces montagnes pour plusieurs raisons. D'une part, elles offraient un abri naturel contre les attaques des tribus hostiles, d'autre part leur paroi est facile à creuser en forme d'abris ou de tombeaux. L'existence de sources a permis parfois l'installation de station-oasis, comme Madā'in Ṣāliḥ/ al-Hiğr الحجر/مدائز صالح, al-'Ulā العلائ ou Tamyā' تيماء en Arabie du Nord-Ouest. Dans le Wādī Iram, on doit à la source de 'Ayn aš-Šallāleh عré la présence d'une agglomération, d'un temple dédié à la déesse arabe al-Lāt اللات ', au pied de la montagne, et d'un sanctuaire rupestre à la source même. 


\section{LES VISITEURS}

Le premier a avoir signalé la présence d'inscriptions à 'Ayn aš-Šallallé, dans le Wādī Iram, a été LAWRENCE d'Arabie, en 1926, dans son livre Les sept piliers de la sagesse, pp. 363-464.

En 1932, M. Georges Horsfield, Directeur des Antiquités dans l'est jordanien, et le Père dominicain SAVIGNAC se rendirent à 'Ayn aš-Šallaleh et alHaz'alī. Ils ont copié de nombreuses inscriptions publiées dans la Revue Biblique de 1932 et 1934. En 1934, ils fouillèrent le temple d'Allāt; le rapport a été publié en 1935, toujours dans la Revue Biblique.

En 1958, Diana KIRKBRIDE et John STRUGnell ont relu les inscriptions de 'Ayn aš-Šallaleh et du temple de Lāt, qu'ils publièrent dans la Revue Biblique de 1960.

En 1947, Lankester HARDing et Enno LiTTMANN effectuent deux courtes missions dans le Wady Iram et ses environs immédiats. Ils relèvent 524 inscriptions qu'ils publient en 1952 dans une ouvrage intitulé Some Thamudic Inscriptions from the Hashimite Kingdom.

Le dernier à avoir exploré le Wādī Iram est le Pr. William JoBLING qui a effectué des prospections de 1980 à 1985, dans le cadre de son exploration générale de la zone 'Aqaba-Ma'ān. Il a publié plusieurs articles préliminaires dans $A D A J\left(\mathrm{n}^{\circ} 25\right.$ à $\left.\mathrm{n}^{\mathrm{o}} 30\right)$, précisant qu'il avait parcouru près de $5000 \mathrm{~km}$ entre 'Aqaba et Ma'ān. Il avait enregistré les inscriptions sur ordinateur mais sa mort prématurée a empêché leur publication.

\section{SiTES}

Les recherches se sont limitées cette année à la région du Wādī Umm Saḥm أم سحم (Carte au 1/50 000, feuilles no 3048 I et 3148 IV) et la zone qui se trouve à l'entrée du Wādī. L'étude des inscriptions du secteur nous permettra d'étudier la distribution géographique des tribus qui y ont nomadisé.

Nous avons choisi cette région pour plusieurs raisons. Elle est d'une part parmi les rares vallées que personne n'a encore prospectées; d'autre part, elle se trouve dans une position stratégique, donnant accès au Wādī Iram aux pasteurs venant de l'Arabie du Nord-Ouest et du Sahl aṣ- Șuwwaneh سهل الصوَّانه (à l'est du Wādī Iram).

Les régions prospectées concernent principalement le Wādī Umm Sạ̣m (3 zones, 15 sites), auxquelles il convient d'ajouter quatre autres zones (8 sites) (Voir carte $n^{\circ}$ 1-2):

1. L'entrée du Wādī Umm Saḥm

2. Le lit du Wādī Umm Saḥm

3. Les affluents du Wād̄̄ Umm Saḥm

4. Sites divers 


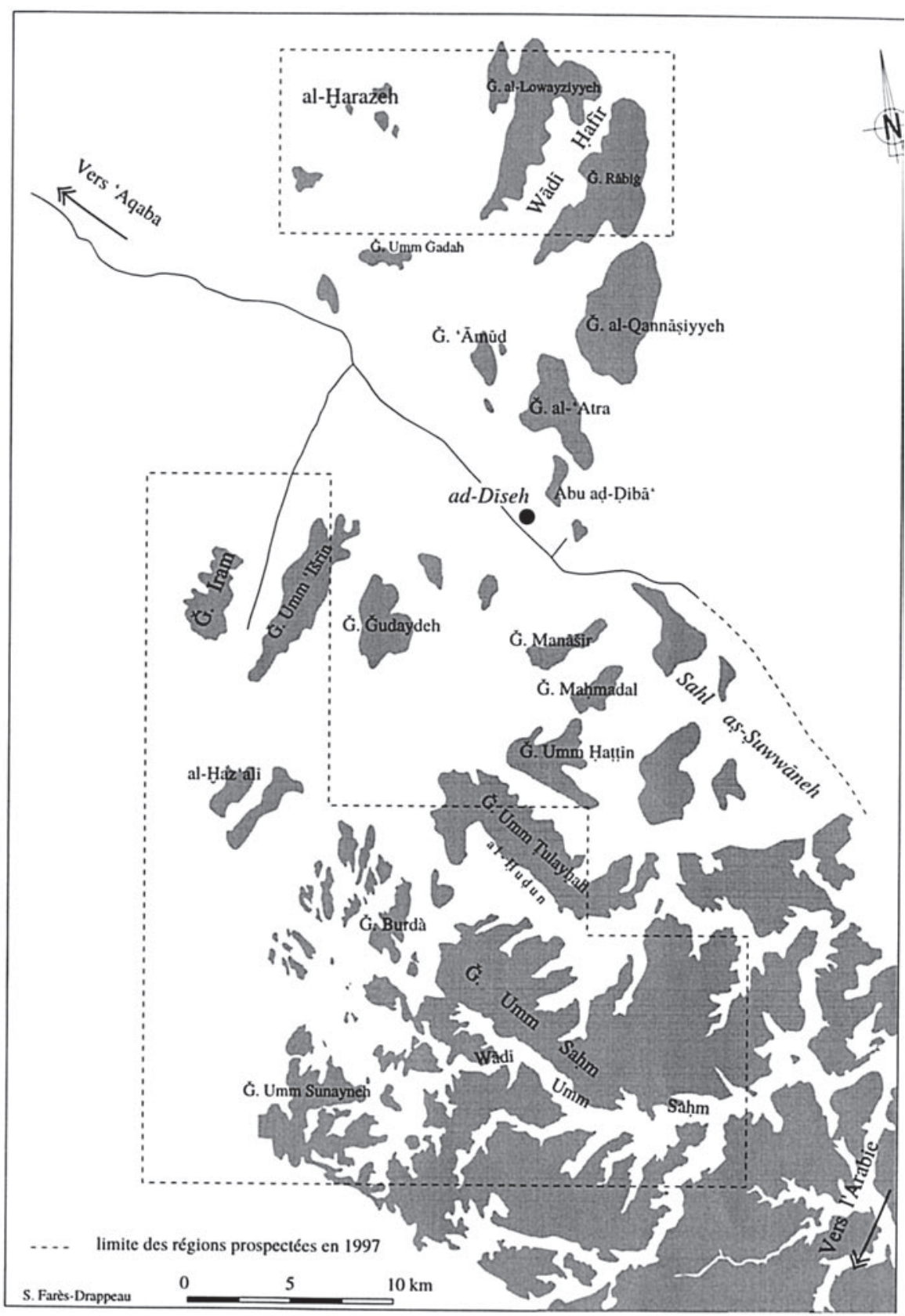

Carte nº 1: l'ensemble des régions prospectées / mission épigraphique dans le Wādī Iram 1997 


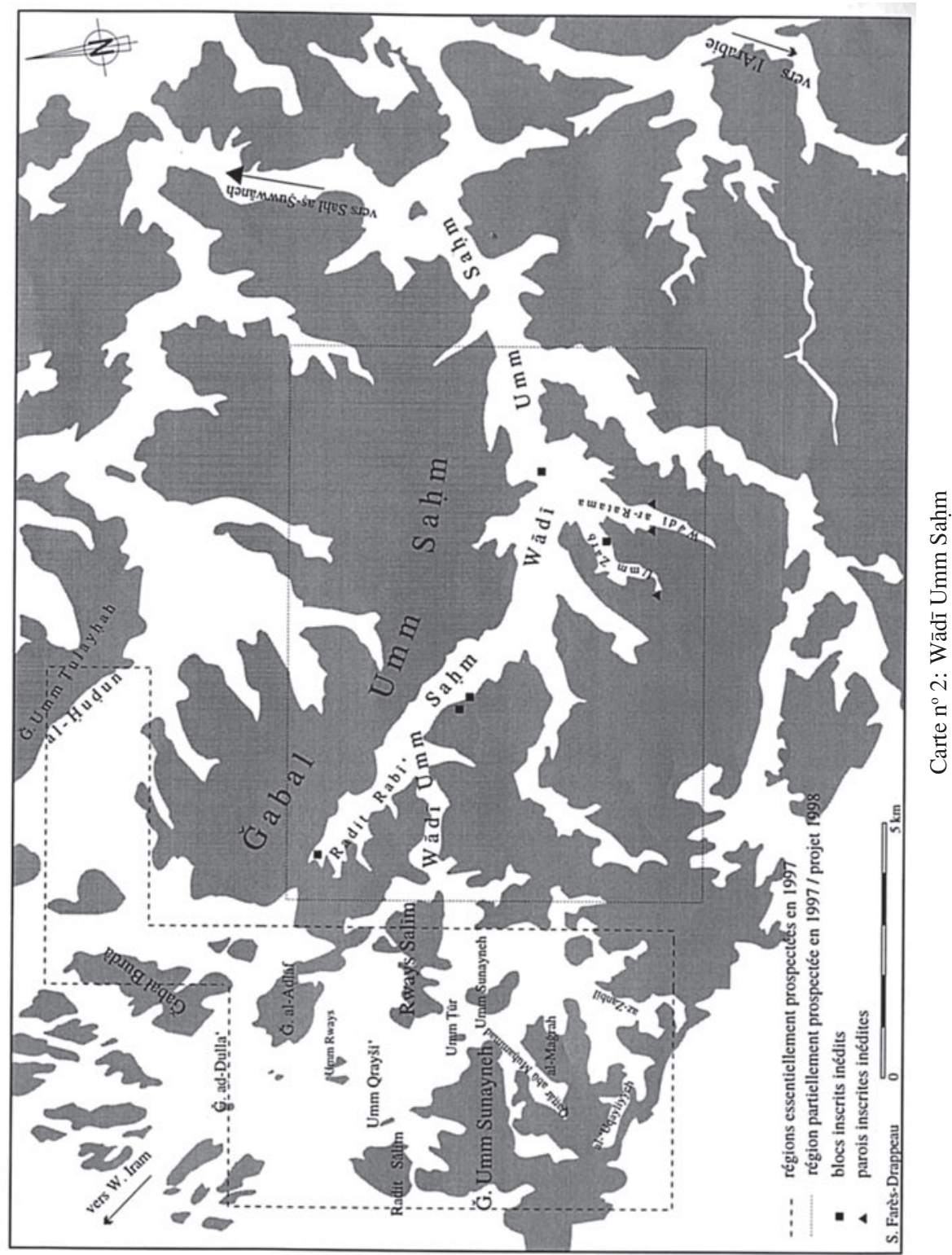

\section{DESCRIPTION DES SITES}

La vallée du Wādī Umm Sahm coule d'ouest en est; longue de $26 \mathrm{~km}$, elle se prolonge en Arabie Séoudite. Elle se trouve à $20 \mathrm{~km}$ au sud-est du Wādī Iram. Constituant un passage naturel entre le Wādī Iram et Sahl aṣ-Ṣuwwāneh d'une part, entre le Wādī Iram et l'Arabie du Nord-Ouest de l'autre, elle est 
l'itinéraire obligé vers ces deux destinations. C'est une vallée de sable, difficilement praticable hors piste, hostile, très pauvre en eau et en végétation. $\mathrm{Au}$ contraire, son débouché qui recueille l'eau de pluie présente une végétation désertique relativement fournie. Des canalisations naturelles se sont formées et les bédouins ont construit partout des barrages afin de récupérer cette eau indispensable. Un réservoir, qui semble antique, porte même des inscriptions de type thamoudéen mentionnant les propriétaires, comme c'était aussi le cas du barrage d'abū aḍ-Dibā' أبو الضباع à ad-Dīsseh الديسه (exploré l'année dernière, au Nord-Est du Wādī Iram) ${ }^{1}$.

\section{III.1. L'entrée du Wādī Umm Sahmm}

Umm Rways رويس سليم Rways Salīm أم رويس se trouvent immédiatement à gauche de l'entrée de la vallée d'Umm Sạ̣m. C'est ici que nous avons relevé le plus grand nombre d'inscriptions. Il est probable que les tribus qui parcouraient l'Arabie du Nord-Ouest dans l'Antiquité y stationnaient. En effet, le lieu offre des commodités naturelles: un abri sous roche et deux grottes. D'ailleurs, les noms des tribus mentionnées par ces textes ne figurent pas ailleurs. On peut donc supposer qu'il s'agissait de tribus nomades installées dans d'autres territoires et qui se rendaient au Wādī Iram pour pratiquer le culte d'Allāt ou pour des échanges commerciaux.

\section{III.2. Le lit du Wādī Umm Saḥm}

Nous avons concentrés nos recherches sur le débouché de ce Wādī et trois bloc très intéressants se sont offerts d'emblée à notre attention. Ils comptent parmi les plus intéressants de cette campagne (avec celui du temple d'Iram). Ils sont situés respectivement:

1. à $1 \mathrm{~km}$ de l'entrée du Wādī, sur la gauche;

2. à $4 \mathrm{~km}$ de l'entrée du Wādī, sur la droite;

3. à $9 \mathrm{~km}$ de l'entrée du Wādī, au milieu du passage.

Ces trois bloc mentionnent des tribus bien attestées en Arabie du Nord telles que 'Ād عاد et Ma'n-'il معن إل.

\section{III.3. Les affluents du Wādī Umm Saḥm}

Les affluents du Wādī sont relativement riches en eau. Leurs vallées constituent des pâturages privilégiés pour les bergers, même si ar-Ratama الرتمه et Umm Zarb أم زئ أمرب sont très difficiles d'accès.

${ }^{1}$ Le l'inscription d'ad-Dīseh a été publiée une première fois par David Graf dans $A D A J$ en 1982, sans que l'auteur se rende compte qu'il s'agissait d'un réservoir d'eau de pluie. La nôtre a montré qu'il s'agissait d'une dédicace de réservoir. Elle a été publiée dans $A D A J, 39,(1995)$, pp. 493-497. 
III.3.1. Ar-Ratama se trouve vers la fin du Wādī, sur la droite. Pour y accéder, il faut emprunter une piste très ensablée au bout de laquelle on doit encore marcher une demi heure pour aboutir à une plate-forme entourée de montagnes dont les parois sont couvertes d'inscriptions et de quelques dessins. Nous avons repéré cinq blocs contenant environ cent inscriptions.

III.3.2. Umm Zarb est une gorge très étroite et profonde, elle se trouve au fond d'un affluent parallèle à ar-Ratama. Parvenus au bout de celui-ci, il nous a fallu une heure de marche pénible et dangereuse, devant parfois escalader la paroi pour pouvoir passer. Un barrage naturel et impressionnant y retenait l'eau de pluie toute l'année. Détruit l'année passée par le violent tremblement de terre qui a secoué la région (nous y étions alors!), il a perdu cette capacité mais, si l'on creuse le sol, l'eau sourd à environ $20 \mathrm{~cm}$ de profondeur. Des inscriptions se trouvent gravées sur les parois voisines, à $2,50 \mathrm{~m}$ de hauteur, accompagnées de dessins de mains (fig. 1). Une abondante concentration

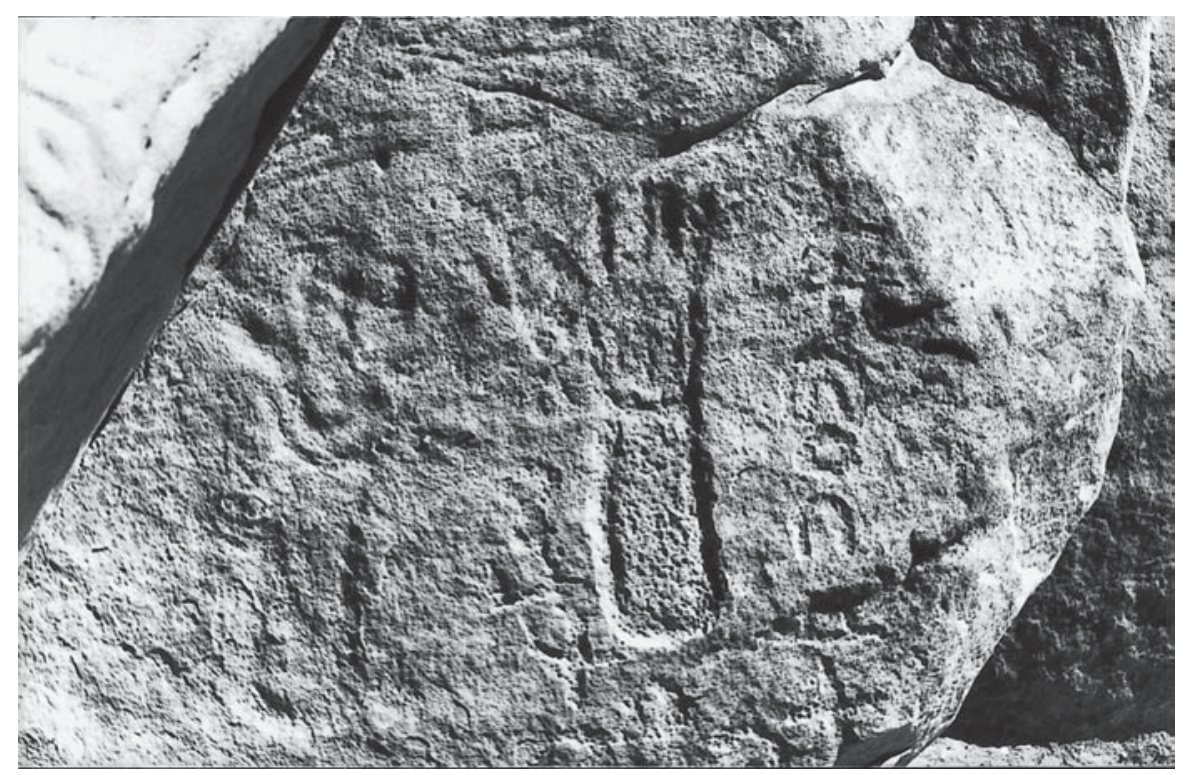

Fig. 1: Dessins de mains.

d'eau en cet endroit dans le passé explique peut-être cette altitude. À l'entrée d'Umm Zarb nous avons trouvé un bloc comportant des inscriptions bien conservées.

III.3.3. Raddit Rabī est un décrochement dans la montagne d'Umm Sahm qui trouve sur la gauche, juste après l'entrée du Wādī Umm Saḥm. Il est large et la piste qui y conduit très bonne. Un bloc inscrit se trouve tout à fait au fond, au pied de la montage. Un texte mentionne Iram comme nom d'homme. C'est la 
deuxième fois que le nom d'Iram se rencontre dans la région (le premier est près du village d'Iram, à Umm al-Quṣayr أم القصير).

\section{III.4. Divers endroits}

\section{III.4.1. Le temple d'Iram}

Des travaux de restauration sont en cours dans le temple d'Iram, dirigés par le Département des Antiquités de Jordanie depuis avril 1997. Ceux-ci ont fait inopinément apparaître une pierre inscrite, posée par les ouvriers sur le mur nord de la chapelle ou cella, et nous l'avons remarquée aussitôt. Le bloc se trouvait auparavant engagé dans le sol de la chapelle où nous avons pu voir en effet son négatif, présentant les mêmes dimensions que lui. La face inscrite s'en trouvait donc jusqu'ici tournée contre terre et soustraite à l'attention des fouilleurs (fig. 2).

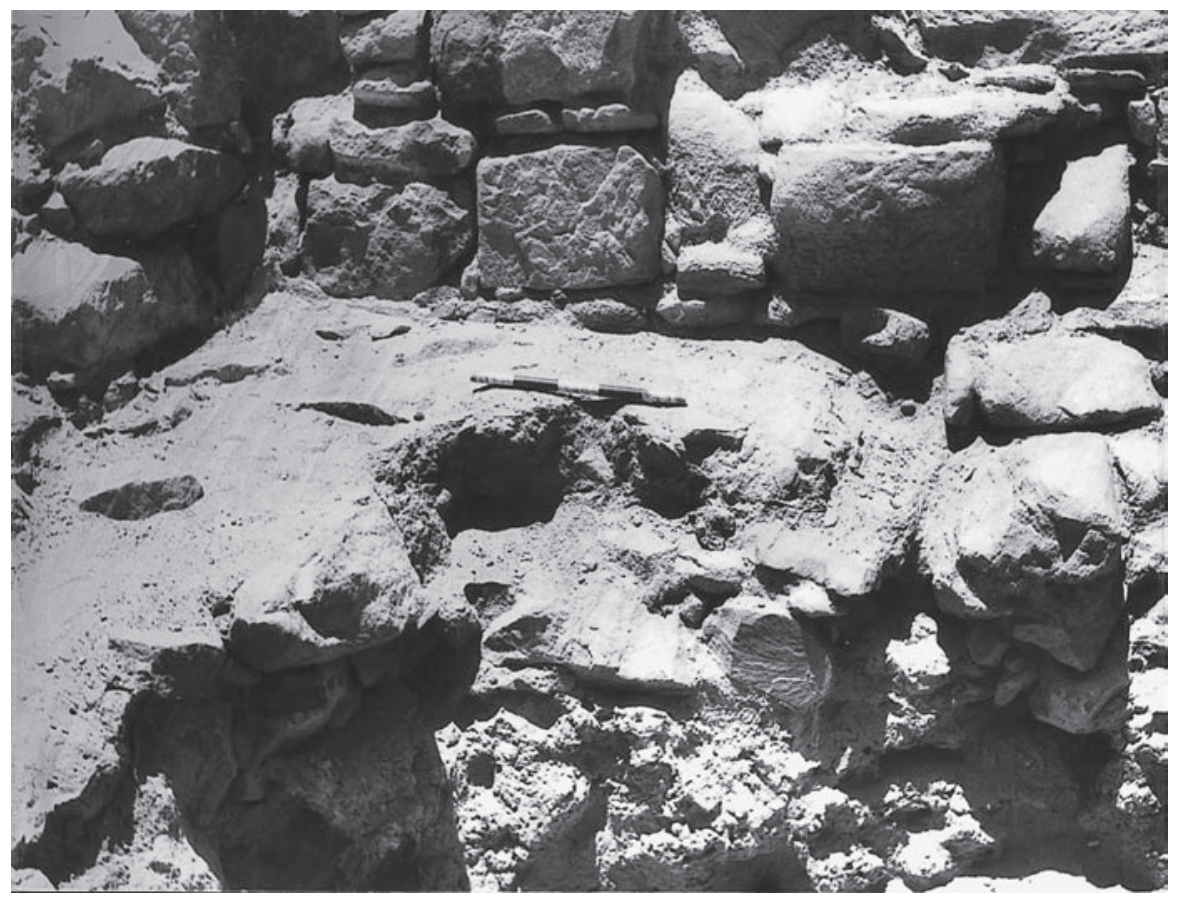

Fig. 2: Emplacement du bloc du Temple d'Iram.

\section{III.4.2. al-Ahayšin}

Ce nom désigne un lieu sis au nord-ouest d'al-Haz'alī الخزعلي. Une inscription nous a été montrée par un bédouin qui cachait le bloc dans le sable. Il a trouvé ce bloc en remploi dans un cimetière. 


\section{III.4.3. Ğabal al-'Utūd}

Cette montagne se trouve au Nord-Ouest du Wādī Iram, bordant la route 'Aqaba / Diseh. Trois inscriptions sont visibles de la route; il s'agit de noms de personnes accompagnés de dessin d'ibex.

\section{III.4.4. al-Harazeh}

Ce nom désigne une montagne et de petites collines situées au nord d'adDīseh. Il y a un barrage nabatéen très connu dans le secteur, décrit par William JOBLING et David GRAF, sur la paroi nord duquel se trouve une inscription nabatéenne datée de l'époque d'Arétas IV. Maurice SARTRE a publié en outre une inscription grecque voisine dans IGLS XXI, IGLJ IV, p. 169.

Les inscriptions que nous avons relevées quant à nous, de type thamoudéen, évoquent la divinité al-Lāt et des scènes de chasses.

\section{LES INSCRIPTIONS}

Les inscriptions présentées ici sont données à titre d'exemple; une étude approfondie est en cours, qui donnera lieu à une publication détaillée.

\section{IV.1. Wādī Iram}

Le bloc bien taillé mesure $55 \mathrm{~cm}$ de long sur $27,5 \mathrm{~cm}$ de large; l'épaisseur est de $23 \mathrm{~cm}$. Le texte en spirale s'étend sur trois lignes (fig. 3 et fac-similé). Le texte est bien conservé.

\section{Transcription}

1 l- $\dot{G} \underline{t} b n$ 'slh bn $\underline{T} k=$

2 mw-bny bt lt

$3 \underline{d}-' l$ 'A $d$

$$
\text { لغث بن أس لاه بن ثم لت }
$$

\section{Lecture}

لغيث بن أوس لاه بن ثكم وبني بيت لات ذو آل عاد

\section{Traduction}

À Gayt, fils de 'Aws-Lāh, fils de Takam, qui a fait construire le sanctuaire d'al-Lāt (pour) le clan 'Ād.

\section{Commentaire}

La tribu 'Ād est connue par le Coran où elle est associée à Thamoud (LIII, 50-52; XLI, 12-13; XIV, 9; XL, 31-32; XXIX, 37; XXII, 43; IX, 70-71). Un 


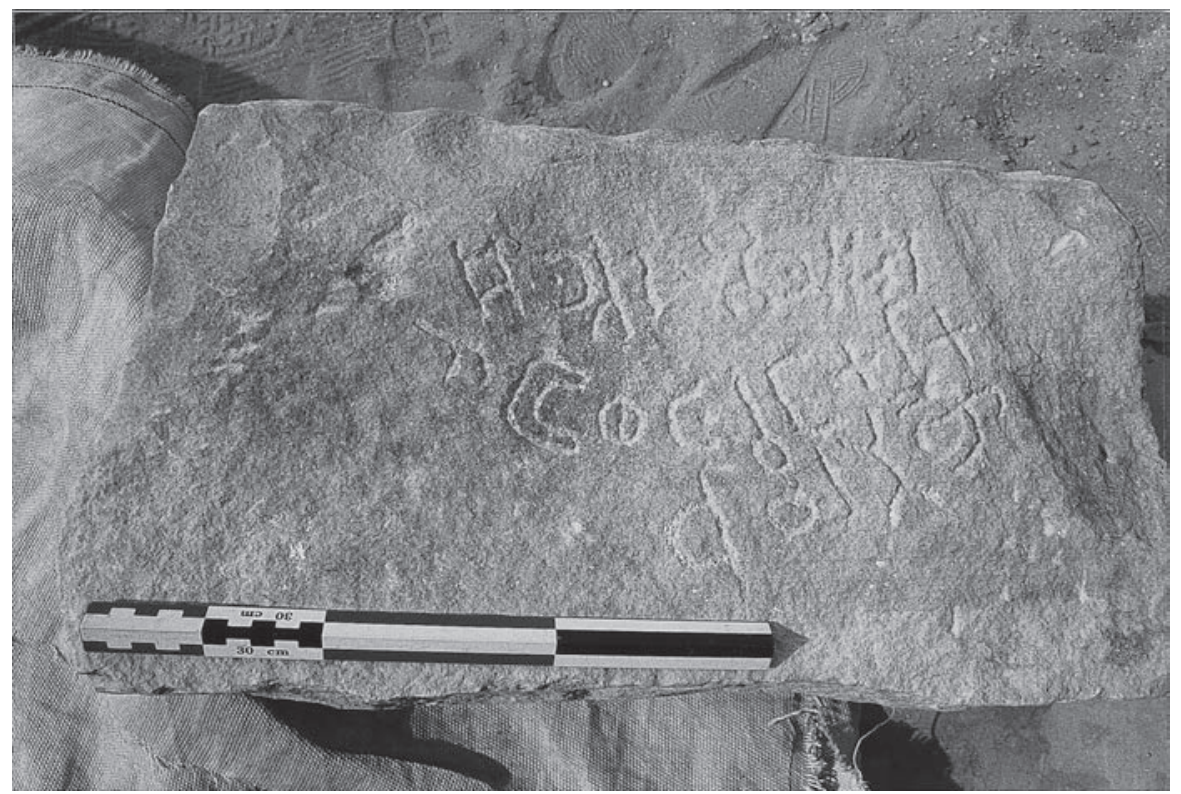

Fig. 3: L'inscription du Temple d'Allāt-Iram avec fac-similé.

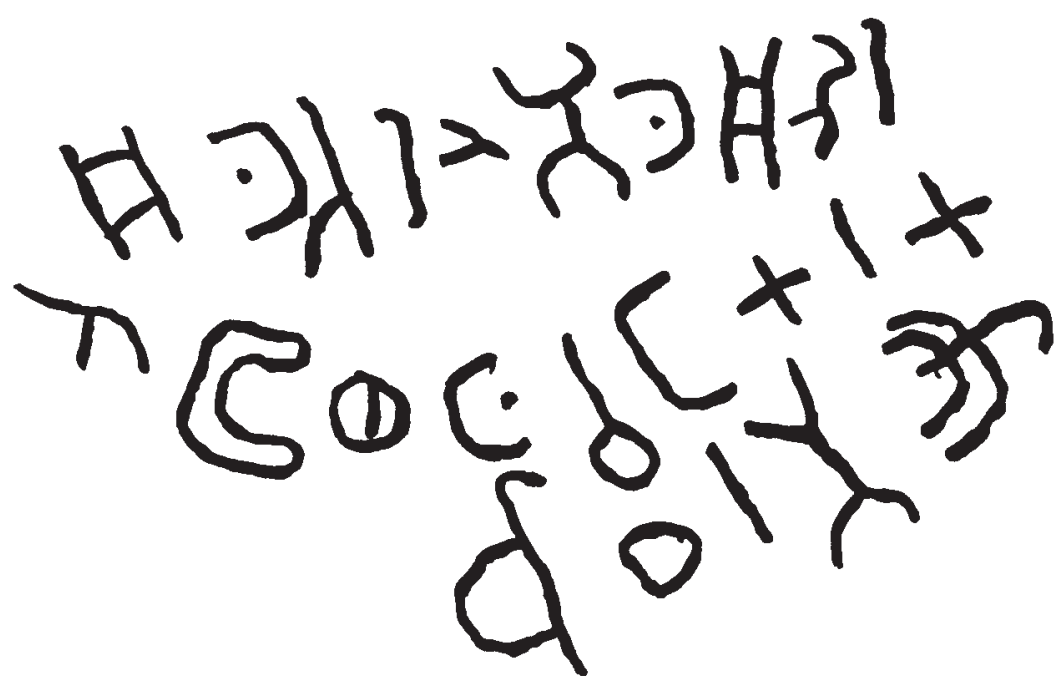

W. Ramm-Temple. Le 10-6-97.

autre verset coranique l'associe à $\operatorname{Iram}^{2}$ ( LXXXIX, 5-7). Le temple du Wādī Iram est connu par une inscription nabatéenne dédicatoire qui se trouve à

2 «Ne vois-tu pas comment ton Seigneur a traité les 'Ad d'Iram, possesseurs de tentes, d'Iram aux colonnes, [peuple tel qu']il n'en fut pas créé de pareil dans le monde?» (R. Blachère, Le Coran, (1949), $\mathrm{N}^{\circ} 42$, t. II, p. 117) 
'Ayn aš-Šallaleh ${ }^{3}$, datée du règne de Rabbel II, entre 70 et 106 ap. J.-C., mais nous n'avions aucun texte relatif à sa construction. Diana KIRKBRIDE, en 1960, établit que le temple fut construit en trois étapes dont la dernière au IIIe s. ap. J.-C. Elle constate la présence d'un village à proximité du temple. Ce village a probablement existé avant le temple mais il doit à celui-ci son développement ${ }^{4}$. La partie de la cella où notre inscription a été découverte n'a jamais été fouillé, KIRKBRIDE l'avait partiellement fouillée. Cette trouvaille laisse supposer, faute de preuve archéologiques, qu'en effet le temple fut initialement bâti par des tribus arabes et cette première fondation serait la cella où nous avons trouvé l'inscription. Cette structure serait ainsi un bayt, nom dont les anciens arabes désignaient leur lieu de culte, sur lequel les nabatéens auraient ensuite greffé leur temple. L'inscription ne donne aucun indice chronologique, néanmoine elle confirme le rôle du temple comme une centre de ralliement des tribus arabes, ce qui a permis le développement du village situé à proximité.

\section{IV.2. al-Ahayšin}

Le bloc, enterré dans le sable par un bédouin, mesure $1,45 \mathrm{~m}$ de long sur $0,30 \mathrm{~m}$ de large; la surface écrite mesure $0,98 \mathrm{~m}$ du long. Les lettres sont bien préservées; il n'y a qu'une seule ligne (fig. 4 et fac-similé). On remarque la présence d'un graffite sur le premier texte; à priori il a été ajouté par la suite.

\section{Transcription}

l-Hrğt bn Wtr bn Hrğt d-'l Hl-'l لخر جت بن وتر بن حر جت ذ آل حل إل

\section{Lecture}

لخر جت بن وتر بن حر جت ذو آل حال إل

\section{Traduction}

À Heriǧat, fils de Watar, fils de Ḩariğat, du clan Hูāl-'il

\section{Commentaire}

Le clan Haall-'îl est attesté dans quatre autres textes relevés durant la mission. Il est probable qu'il comptait parmi les plus notables des groupes nomadisant dans le sud de la Jordanie. Le nom Hrğt est attesté surtout en safaïtique quant au nom Wtr est attesté surtout en thamoudéen (Caskel, Index).

\section{IV.3. Rways Salīm}

Un bloc situé près d'un abri sous roche, juste à droite de l'entrée du Wādī Umm Sạ̣m comporte 18 textes en bon état de conservation, gravés avec des

\footnotetext{
3 Savignac, «Le sanctuaire d'Allat à Iram (1)», RB XLII, (1933), pp; 407-411.

4 Kirkbride, «Le temple nabatéen de Ramm», RB LXVII, (1960), p. 74.
} 


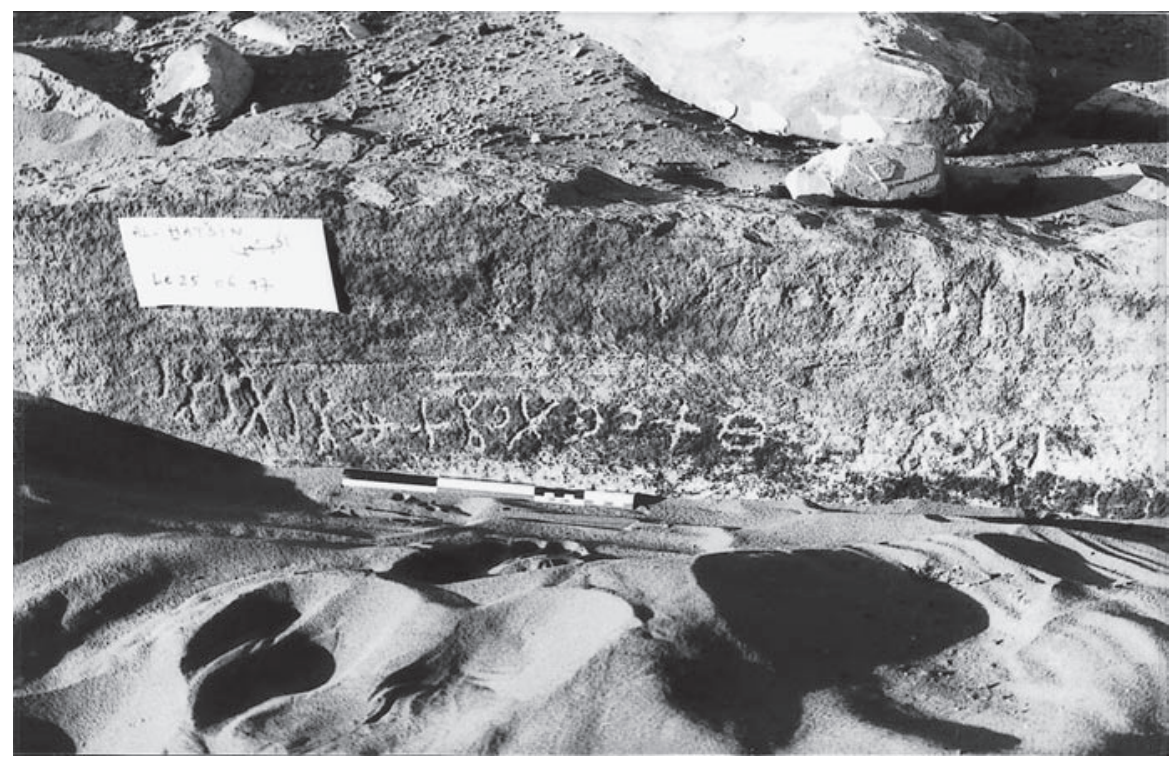

Fig. 4: L’inscription d'al-Ahayšin avec fac-similé.

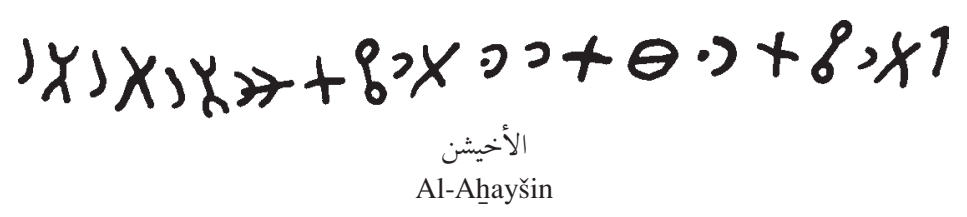

caractère réguliers. La paroi de l'abri sous roche est également couverte d'inscriptions mais certaine sont abîmées. Le bloc présente une inscription mentionnant un clan attesté à plusieurs reprises dans les textes relevés durant la mission. Le texte est gravé de haut en bas (fig. 5 et fac-similé).

\section{Transcription}

l-'bd bn Mḥlb d-'l Mzn

$$
\text { لعبد بن محلب ذذ آل مزن }
$$

\section{Lecture}

لعبد بن محلب ذو آل مزن

Transcription

À 'Abd fils de Mihlib du clan de Mazan.

\section{Commentaire}

Une tribu portant le nom de Mazan existe de nos jours dans la région d'adDīseh. Son nom est prononcé par les bédouins Mzinah. Le nom ' $b d$ dont la signification est esclave est un nom très répondu chez les arabes. Le nom Mhllb 


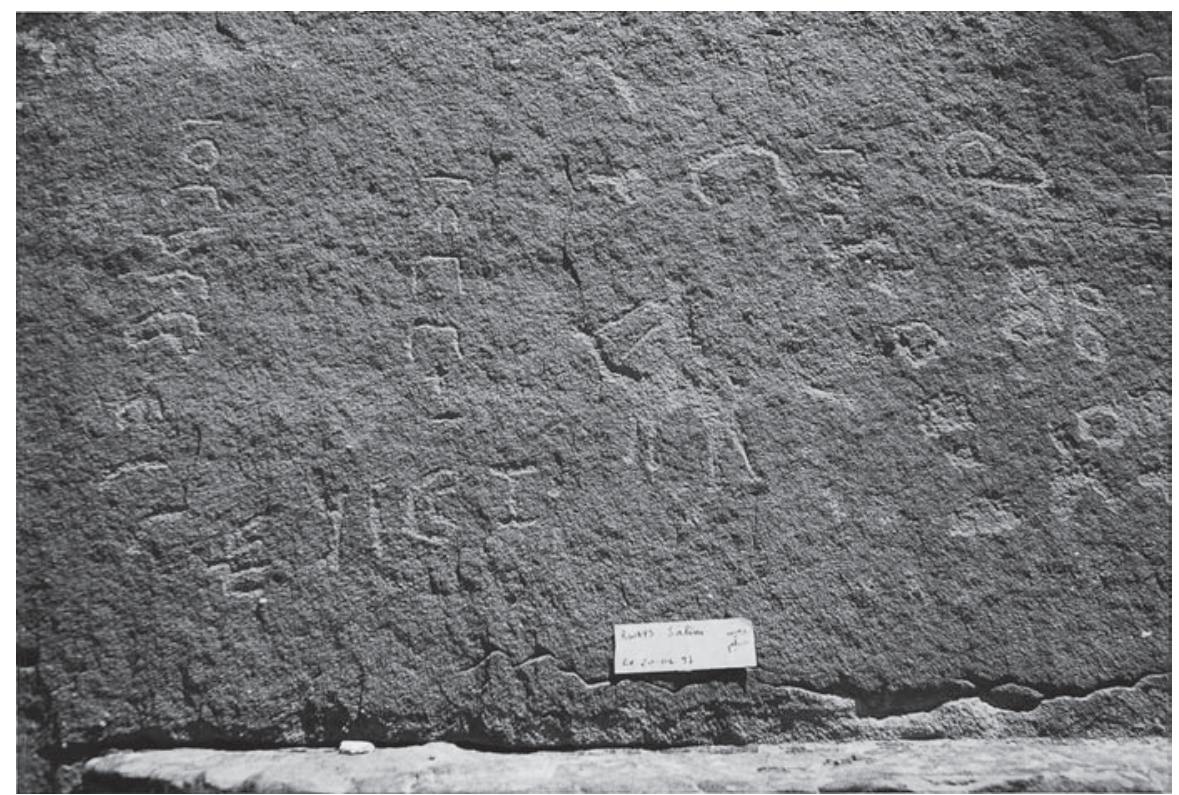

Fig. 5: L'inscription d'Um Rways avec fac-similé.

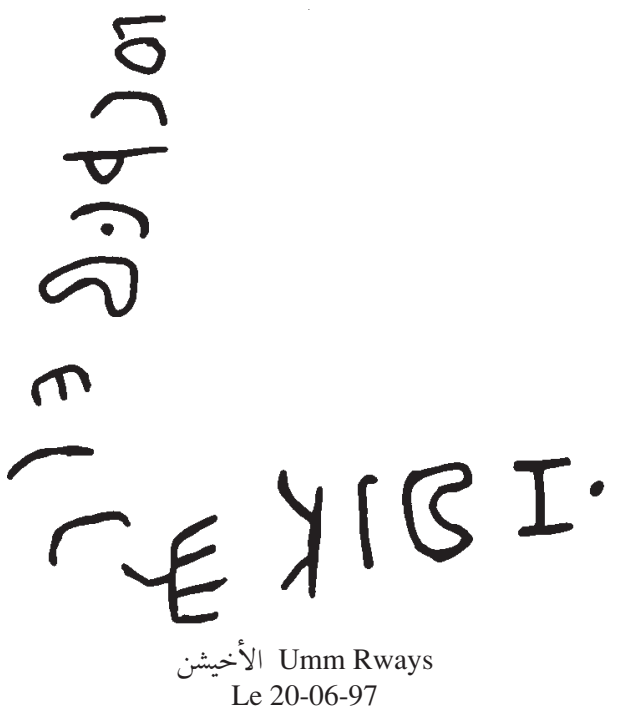

peut avoir plusieurs significations, selon la vocalisation, ainsi Mihlab a pour signification le vase dans lequel on trait, Mahlab signifie celui qui aide à traire. Ce nom est nouveau.

\section{IV.4. Wādī Umm Sahm}

Le bloc inscrit se trouve au pied de la montage, il comporte 34 textes. Des graffitis récents s'y trouvent aussi et plusieurs Wasm. Les textes sont bien conser- 
vés. Le texte que nous donnons ici est gravé en boustrophédon (fig. 6 et facsimilé).

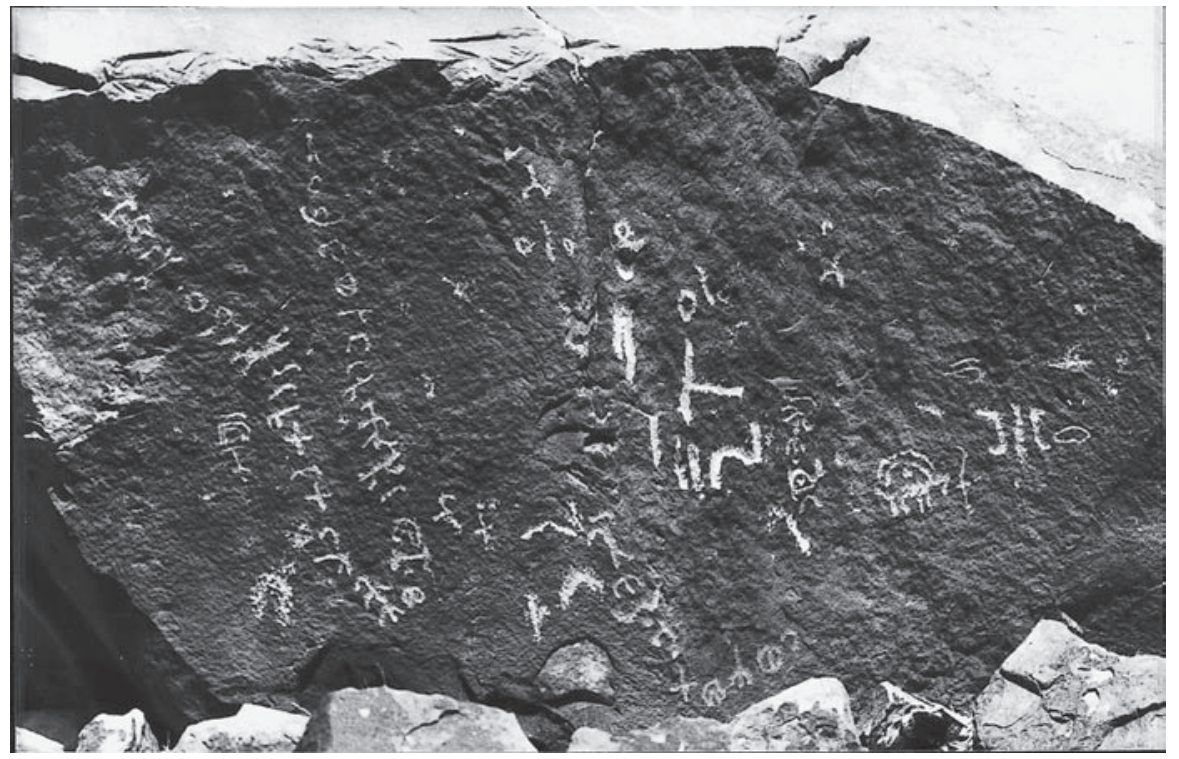

Fig. 6: L'inscription d'Um Sahm avec fac-similé.

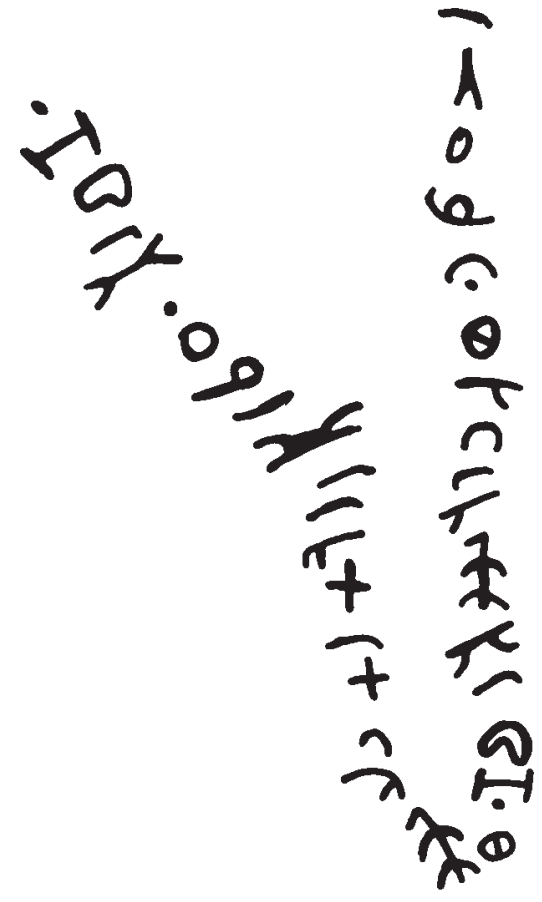

Umm Saḥm 


\section{Transcription}

l-S'd bn Whb-Lh d-'l Mzn w-zkrt Lt kll 'šy'n 'l Mzn

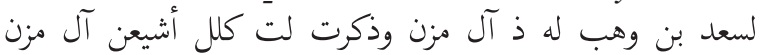

\section{Lecture}

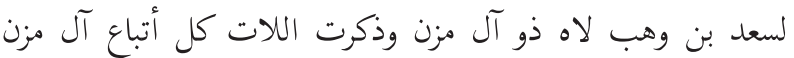

Traduction

À Sa'd fils, de Wahb-Lāh, du clan Mazan, que la divinité al-Lāt se souvienne de tous les membre du clan Mazan!

\section{Commentaire}

Dans ce texte, nous avons le premier emploi du terme 'šy' qui désigne les compagnons, les partisans. En arabe, le mot désigne une réunion d'hommes suivant le même chef ${ }^{5}$. Il est probable qu'il s'agisse ici d'une confédération tribale dont Mazan مزن est la famille chef. Ce mot est attesté surtout en safaïtique.

\section{BIBLIOGRAPHIE}

BlachÈRE, R., Le Coran, (1949).

FARÈs-DRAPPEAU, Saba, «Inscriptions de type dédanite de Abū aḍ-Dibā'», ADAJ 39, (1995), pp. 493-498.

GRAF, David, «Dedanite and Minaean Inscriptions from the Hisma», ADAJ 27, (1983), pp. 555-567.

Grimme, Hubert, «A propos de quelques graffites du temple de Iram», Revue Biblique 45, (1936), pp. 90-95.

HaRding, Lankester, Some Thamudic Inscriptions from the Hashimite Kingdom of the Jordan, (Leiden, 1952).

Jobling, William, «Aqaba-Ma'an Survey, Jan-Feb, 1981», ADAJ 26, (1982), pp. 199209.

—, «The 1982 Archaeological and Epigraphic Survey of the 'Aqaba-Ma'an Area of Southern Jordan», ADAJ 27, (1983), pp. 185-196.

—, «Preliminary Report on the Forth Season of the 'Aqaba-Ma'an Archaeological and Epigraphic Survey 1982/1983», ADAJ 27, (1983), pp. 197-208.

—, «The Fifth Season of the Aqaba-Ma'an Survey 1984», ADAJ 28, (1984), pp. 191202.

-, «Preliminary Report of the Sixth Season of the Aqaba-Ma'an Epigraphic and Achaeological Survey», ADAJ 29, (1985), pp. 211-220.

-, «North Arabian (Thamudic) Inscriptions and Rock Art from the Aqaba-Ma'an Area of Southern Jordan», ADAJ 30, (1986), pp. 261-284.

KIRKBRIDE, Diana, «Le temple nabatéen de Iram. Son évolution architecturale», Revue Biblique, 67, (1960-, pp. 65-92, pl. III-IX

${ }^{5}$ Kazimirski, tome I. 
Lawrence, T. D., The Seven Pillars of Wisdom, (London, 1926), pp. 363-464.

SARTRE, M., Inscriptions de la Jordanie, IGLS 21-IGLJ 4, (BAH 115, Beyrouth, 1993). SAvignac, R., «Note de voyage, Le sanctuaire d'Allat à Iram», Revue Biblique 41, (1932), pp. 581-597.

- «Le sanctuaire d'Allat à Iram», Revue Biblique 42, (1933), pp. 405-422.

- «Le sanctuaire d'Allat à Iram (suite)», Revue Biblique 43, (1934), pp. 572-589 [Appendice de Gonzague Ryckmans «Inscriptions minéennes de Ramm».

Savignac, R, Horsfield, G, «Le Temple de Ramm», Revue Biblique 44, (1935), pp. 245-278. 